\title{
Spectroscopic properties of the B meson
}

\author{
Nayneshkumar Devlani ${ }^{1, a}$, Virendrasinh H. Kher ${ }^{1, b}$, and Ajay Kumar Rai ${ }^{2, c}$ \\ ${ }^{1}$ Applied Physics Department, Polytechnic, The M.S. University of Baroda, Vadodara 390002, INDIA \\ ${ }^{2}$ Department of Applied Physics , S.V. National Institute of Technology, Surat 395007, INDIA
}

\begin{abstract}
Investigation of the $B(b \bar{q}) ; q=u, d$ meson properties is carried out using variational method within phenomenological quark antiquark potential(coulomb plus power) model using the Gaussian wave function. $O(1 / m)$ correction to the potential energy term and relativistic corrections to the kinetic energy term of the hamiltonian are incorporated. Spin-orbit, spin-spin and tensor interactions are employed to obtain the mass spectra. Various other properties such as the decay constants, e1 and $\mathrm{m} 1$ transitions are also obtained
\end{abstract}

\section{Introduction}

Potential models based on the asymptotic freedom and the confinement features of QCD have been quite successful in the study of mesons composed of quarks which are heavy. The $B(b \bar{q}) ; q=u, d$, consists of a heavy and a light quark[1]. Therefore the validity of a potential model approach for such a system should be judged by its predictions. The $B$ meson has been extensively studied in various theoretical schemes including potential models[2-7]. In general there is a fair agreement between the experimentally measured ground state masses and the various models. However for the $L=11^{3} P_{0}$ state one finds significant differences.

In this paper we employ a conventional potential model scheme that incorporates kinematic relativistic corrections to the kinetic energy term as well as $O(1 / \mathrm{m})$ corrections to the potential energy term[8]. The paper is organized as following. In section 2 theoretical formulation as well as results for the mass spectra are outlined. Subsequently in section 3 decay constants and in section 4 electromagnetic transition widths are obtained. Finally the conclusion is made in section 5.

\section{Theoretical formulation}

For the study of the $B$ meson we consider the relativistic Hamiltonian in which motion of the quarks inside the meson is relativistic[9-11]

$$
H=\sqrt{\mathbf{p}^{2}+m_{b}^{2}}+\sqrt{\mathbf{p}^{2}+m_{\bar{q}}^{2}}+V(\mathbf{r})
$$

\footnotetext{
ae-mail: nayneshdev@gmail.com

be-mail: vhkher@yahoo.co.in

ce-mail: raiajayk@gmail.com
} 
where $\mathbf{p}$ is the relative momentum of the quark-antiquark and $m_{b}$ is the $b$ quark mass and $m_{\bar{q}}$ is the $q=u, d$ quark mass. The Hamiltonian in $\mathrm{Eq}(1)$ represents the energy of the meson in the meson rest frame. We expand the kinetic energy(K.E.) part of the Hamiltonian, as

$$
K . E .=\frac{\mathbf{p}^{2}}{2}\left(\frac{1}{m_{b}}+\frac{1}{m_{\bar{q}}}\right)-\frac{\mathbf{p}^{4}}{8}\left(\frac{1}{m_{b}^{3}}+\frac{1}{m_{\bar{q}}^{3}}\right)+O\left(p^{6}\right)
$$

and $V(\mathbf{r})$ is the quark-antiquark potential[12],

$$
V(r)=V^{(0)}(r)+\left(\frac{1}{m_{b}}+\frac{1}{m_{\bar{q}}}\right) V^{(1)}(r)+O\left(\frac{1}{m^{2}}\right) ;
$$

where[13-15],

$$
V^{(0)}(r)=-\frac{\alpha_{c}}{r}+A r+V_{0}
$$

$A$ is the potential parameter and $V_{0}$ is a constant. $\alpha_{c}=(4 / 3) \alpha_{S}\left(M^{2}\right), \alpha_{S}\left(M^{2}\right)$ is the strong running coupling constant. The non-perturbative form of $V^{(1)}(r)$ is not yet known, but leading order perturbation theory yields

$$
V^{(1)}(r)=-C_{F} C_{A} \alpha_{s}^{2} / 4 r^{2}
$$

where $C_{F}=4 / 3$ and $C_{A}=3$ are the Casimir charges of the fundamental and adjoint representation, respectively[12]. The value of the QCD coupling constant $\alpha_{s}\left(M^{2}\right)$ is determined through the simplest model with freezing $[16,17]$, namely

$$
\alpha_{s}\left(M^{2}\right)=\frac{4 \pi}{\left(11-\frac{2}{3} n_{f}\right) \ln \frac{M^{2}+M_{B}^{2}}{\Lambda^{2}}}
$$

where $M=2 m_{Q} m_{\bar{q}} /\left(m_{Q}+m_{\bar{q}}\right), M_{B}=0.95 \mathrm{GeV}[16,17]$, and $\Lambda=0.413 \mathrm{GeV}[18]$.

We have used the gaussian wave function in the present study. The gaussian wave function in position space has the form

$$
R_{n l}(\mu, r)=\mu^{3 / 2}\left(\frac{2(n-1) !}{\Gamma(n+l+1 / 2)}\right)^{1 / 2}(\mu r)^{l} e^{-\mu^{2} r^{2} / 2} L_{n-1}^{l+1 / 2}\left(\mu^{2} r^{2}\right)
$$

and in momentum space has the form

$$
R_{n l}(\mu, p)=\frac{(-1)^{n}}{\mu^{3 / 2}}\left(\frac{2(n-1) !}{\Gamma(n+l+1 / 2)}\right)^{1 / 2}\left(\frac{p}{\mu}\right)^{l} \times e^{-p^{2} / 2 \mu^{2}} L_{n-1}^{l+1 / 2}\left(\frac{p^{2}}{\mu^{2}}\right)
$$

Here, $\mu$ is the variational parameter and $L$ is Laguerre polynomial.

For the present study, we employ the Ritz variational scheme. We obtain the expectation values of the Hamiltonian as

$$
H \psi=E \psi
$$

The variational parameter, $\mu$ is determined for each state using the Virial theorem[19]. Gaussian wavefunction in position space has been employed to obtain the expectation value of the potential energy part in the Virial theorem while momentum space wave function has been used to obtain the kinetic energy part. 
Table 1. Spin averaged masses of $B$ meson.

\begin{tabular}{ccccccccc}
\hline \multirow{2}{*}{ State } & \multirow{2}{*}{$\mu(\mathrm{GeV})$} & \multirow{2}{*}{$|R(0)|\left(\mathrm{GeV}^{3 / 2}\right)$} & \multicolumn{5}{c}{$M_{S A}(\mathrm{GeV})$} \\
\cline { 4 - 8 } & & Present work & Expt.[1] & {$[2]$} & {$[7]$} & {$[4]$} & {$[3]$} \\
\hline 1S & 0.494 & 0.521 & 5.314 & 5.314 & 5.314 & 5.313 & 5.318 & 5.313 \\
2S & 0.317 & 0.218 & 5.878 & & 5.902 & 5.842 & 5.860 & 5.912 \\
3S & 0.260 & 0.145 & 6.329 & 6.385 & 6.131 & 6.232 & 6.340 \\
4S & 0.231 & 0.112 & 6.731 & 6.785 & 6.347 & & \\
1P & 0.340 & & 5.758 & 5.745 & 5.696 & 5.695 & 5.717 \\
2P & 0.270 & & 6.210 & 6.249 & 6.030 & 6.105 & 6.184 \\
1D & 0.297 & & 6.036 & 6.106 & 5.924 & 5.970 & 6.007 \\
2D & 0.251 & & 6.448 & 6.540 & 6.183 & & 6.306 \\
\hline
\end{tabular}

As the interaction potential assumed here does not contain the spin dependent part, $\operatorname{Eq}(9)$ gives the spin averaged masses of the system. The calculated spin averaged mass of the ground state is matched with the experimental spin-averaged mass using the equation [15]

$$
M_{S A}=M_{P}+\frac{3}{4}\left(M_{V}-M_{P}\right)
$$

; where $M_{V}$ and $M_{P}$ are the vector and pseudoscalar meson ground state masses taken from ref [1]. This fixes the parameter $V_{0}$. Using this value of $V_{0}$ we calculate $S, P$, and $D$ wave spin-averaged masses of $B$ mesons which are listed in Table 1 . For the comparison for the $n J$ state, we compute the spin-averaged or the center of weight mass from the respective theoretical values as [15]

$$
M_{C W, n}=\frac{\Sigma_{J} 2(2 J+1) M_{n J}}{\Sigma_{J} 2(2 J+1)}
$$

where, $M_{C W, n}$ denotes the spin-averaged mass of the $n$ state and $M_{n J}$ represents the mass of the meson in the $n J$ state.

The value of the radial wave function $R(0)$ for $0^{-+}$and $1^{--}$states would be different due to their spin dependent hyperfine interaction. The spin hyperfine interaction of the heavy-light flavored mesons is small and this can cause a small shift in the value of the wave function at the origin[15, 20].

The parameters used to calculate the low lying masses of the $B$ meson are $A=0.1 \mathrm{GeV}^{-1}, m_{\bar{q}}=$ $0.45 \mathrm{GeV}, m_{b}=4.88 \mathrm{GeV}$ and the value of the constant $V_{0}=-0.132 \mathrm{GeV}$. The spin averaged masses for $\mathrm{S}, \mathrm{P}$ and $\mathrm{D}$ states are tabulated in Table 1. It can be observed that the spin-averaged masses obtained are in good agreement with experimental and other theoretical predictions.

\subsection{Excited states}

We add separately (in Eq.(9)) the spin-dependent part of the usual one gluon exchange potential (OGEP) between the quark anti quark for computing the hyperfine and spin-orbit shifting of the lowlying $S, P$ and $D$-states. Thus to take into account the spin dependent and spin-orbit interaction, causing the splitting of the $n L$ levels one introduces additional term in the Hamiltonian[21-23]

$$
\begin{aligned}
V_{S D}(\mathbf{r})= & \left(\frac{\mathbf{L} \cdot \mathbf{S}_{\mathbf{b}}}{2 m_{b}^{2}}+\frac{\mathbf{L} \cdot \mathbf{S}_{\overline{\mathbf{c}}}}{2 m_{\bar{c}}^{2}}\right)\left(-\frac{d V^{(0)}(r)}{r d r}+\frac{8}{3} \frac{\alpha_{S}}{r^{3}}\right)+\frac{4}{3} \frac{\alpha_{S}}{m_{b} m_{\bar{c}}} \frac{\mathbf{L} \cdot \mathbf{S}}{r^{3}}+\frac{8 \alpha_{S}}{9 m_{b} m_{\bar{c}}} \mathbf{S}_{\mathbf{b}} \cdot \mathbf{S}_{\overline{\mathbf{c}}} 4 \pi \delta(\mathbf{r}) \\
& +\frac{4}{3} \alpha_{S} \frac{1}{m_{b} m_{\bar{c}}}\left\{3\left(\mathbf{S}_{\mathbf{b}} \cdot \mathbf{n}\right)\left(\mathbf{S}_{\overline{\mathbf{c}}} \cdot \mathbf{n}\right)-\left(\mathbf{S}_{\mathbf{b}} \cdot \mathbf{S}_{\overline{\mathbf{c}}}\right)\right\} \frac{1}{r^{3}}, \quad \mathbf{n}=\frac{\mathbf{r}}{r}
\end{aligned}
$$


where $V^{(0)}(r)$ is the phenomenological potential, the first terms takes into account the relativistic corrections to the potential $V(r)$, the second term accounts spin orbital interaction, third term is usual spin-spin interaction part which is responsible for pseudoscalar and vector meson splitting(Eq. (15) $\&(16))$ and fourth term stands for tensor interaction.

In the case of quark and antiquark of unequal mass charge-conjugation parity is no longer a good quantum number and so the states with $J=L$, are mixtures of spin-triplet $\left.\left.\right|^{3} L_{L}\right\rangle$ and spin-singlet $\left|{ }^{1} L_{L}\right\rangle$ states: $J=L=1,2,3, \ldots$

$$
\begin{aligned}
& \left|\psi_{J}\right\rangle=\left|{ }^{1} L_{L}\right\rangle \cos \phi+\left|{ }^{3} L_{L}\right\rangle \sin \phi \\
& \left|\psi_{J}^{\prime}\right\rangle=-\left|{ }^{1} L_{L}\right\rangle \sin \phi+\left|{ }^{3} L_{L}\right\rangle \cos \phi
\end{aligned}
$$

where $\phi$ is the mixing angle and the primed state has the heavier mass. Such mixing occurs due to the nondiagonal spin-orbit and tensor terms in Eq (9). The masses of the physical states were obtained by diagonalizing the mixing matrix. The calculated values of the mass spectra of $B$ meson are listed in Table 2. We are following spectroscopic notation $n^{2 S+1} L_{J}$ in Table 2. Overall the mass spectrum is in satisfactory agreement with experimental as well as other theoretical predictions.

\begin{tabular}{|c|c|c|c|c|c|c|c|c|}
\hline State & This Work & Expt.[1] & {$[24]$} & [7] & [4] & [3] & {$[6]$} & {$[25]$} \\
\hline $1^{1} S_{0}$ & 5.264 & 5.279 & 5.280 & 5.277 & 5.280 & 5.279 & 5.279 & 5.279 \\
\hline $1^{3} S_{1}$ & 5.330 & 5.325 & 5.326 & 5.325 & 5.330 & 5.324 & 5.324 & 5.325 \\
\hline $1^{3} P_{0}$ & 5.729 & $5.732_{ \pm 0.02}^{ \pm 0.005}$ & 5.749 & 5.678 & 5.650 & 5.706 & 5.689 & 5.795 \\
\hline $1 P_{1}^{\prime}$ & 5.757 & & 5.774 & 5.699 & 5.690 & 5.742 & 5.744 & 5.859 \\
\hline $1 P_{1}$ & 5.755 & 5.723 & 5.723 & 5.686 & 5.690 & 5.700 & 5.731 & 5.839 \\
\hline $1^{3} P_{2}$ & 5.766 & 5.743 & 5.741 & 5.704 & 5.710 & 5.714 & 5.759 & 5.875 \\
\hline $2^{1} S_{0}$ & 5.870 & & 5.890 & 5.822 & 5.830 & 5.886 & 5.892 & \\
\hline $2^{3} S_{1}$ & 5.881 & & 5.906 & 5.848 & 5.870 & 5.920 & 5.924 & \\
\hline $1^{3} D_{1}$ & 6.069 & & 6.119 & 6.005 & 5.970 & 6.025 & & \\
\hline $1 D_{2}^{\prime}$ & 6.077 & & 6.121 & 5.955 & 5.980 & 6.037 & & \\
\hline $1 D_{2}$ & 6.007 & & 6.103 & 5.920 & 5.960 & 5.985 & & \\
\hline $1^{3} D_{3}$ & 6.013 & & 6.091 & 5.871 & 5.970 & 5.993 & & \\
\hline $2^{3} P_{0}$ & 6.195 & & 6.221 & 6.010 & 6.060 & 6.163 & & \\
\hline $2 P_{1}$ & 6.206 & & 6.209 & 6.022 & 6.100 & 6.175 & & \\
\hline $2 P_{1}^{\prime}$ & 6.214 & & 6.281 & 6.028 & 6.100 & 6.194 & & \\
\hline $2^{3} P_{2}$ & 6.213 & & 6.260 & 6.040 & 6.120 & 6.188 & & \\
\hline $3^{1} S_{0}$ & 6.326 & & 6.379 & 6.117 & 6.210 & 6.320 & & \\
\hline $3^{3} S_{1}$ & 6.331 & & 6.387 & 6.136 & 6.240 & 6.347 & & \\
\hline $2^{3} D_{1}$ & 6.425 & & 6.534 & 6.248 & 6.240 & & & \\
\hline $2 D_{2}^{\prime}$ & 6.482 & & 6.554 & 6.207 & 6.320 & & & \\
\hline $2 D_{2}^{2}$ & 6.476 & & 6.528 & 6.179 & 6.310 & & & \\
\hline $2^{3} D_{3}$ & 6.429 & & 6.542 & 6.140 & 6.320 & & & \\
\hline $4^{1} S_{0}$ & 6.729 & & 6.781 & 6.335 & 6.520 & & & \\
\hline $4^{3} S_{1}$ & 6.732 & & 6.786 & 6.351 & & & & \\
\hline
\end{tabular}

Table 2. Masses of the B mesons(in GeV). 


\section{Decay constants}

The decay constants of mesons are important parameters in the study of leptonic or non-leptonic weak decay processes. In the non-relativistic limit, we compute the decay constants using the Van-RoyenWeisskopf formula[26],

$$
f_{P / V}^{2}=\frac{12\left|\psi_{P / V}(0)\right|^{2}}{M_{P / V}} \bar{C}^{2}\left(\alpha_{S}\right) ;
$$

where $\bar{C}\left(\alpha_{S}\right)$ is the QCD correction factor given by[27]

$$
\bar{C}^{2}\left(\alpha_{S}\right)=1-\frac{\alpha_{S}}{\pi}\left[2-\frac{m_{Q}-m_{\bar{q}}}{m_{Q}+m_{\bar{q}}} \ln \frac{m_{Q}}{m_{\bar{q}}}\right] .
$$

The computed $f_{P}$ and $f_{V}$ for $B$ meson using equation (15) are tabulated in Table 3 . The Eq. (15) also gives the inequality[19]

$$
\sqrt{m_{v}} f_{v} \geq \sqrt{m_{p}} f_{p}
$$

Our results are in accordance with Eq. (17).

Table 3. Decay constants of the $B$ meson(in $\mathrm{GeV}$ ).

\begin{tabular}{cccccc}
\hline & & $1 \mathrm{~S}$ & $2 \mathrm{~S}$ & $3 \mathrm{~S}$ & $4 \mathrm{~S}$ \\
\hline & This work & 0.219 & 0.088 & 0.056 & 0.042 \\
& {$[28]$} & $0.191 \pm 0.009$ & & & \\
$f_{P}$ & {$[29]$} & $0.197 \pm 0.009$ & & & \\
& {$[30]$} & $0.193 \pm 0.011$ & & & \\
& {$[31]$} & $0.198 \pm 0.014$ & & & \\
\hline \multirow{4}{*}{$f_{V}$} & This work & 0.221 & 0.088 & 0.056 & 0.042 \\
& {$[32]$} & $0.190_{-0.027}^{+0.028}$ & & & \\
& {$[33]$} & 0.190 & & & \\
& {$[34]$} & 0.164 & & & \\
& {$[35]$} & $0.194 \pm 0.008$ & & & \\
\hline
\end{tabular}

\section{Electromagnetic transition widths}

\subsection{Electric Dipole Transition}

The radiative widths are calculated in the dipole approximation. The E1 matrix elements are determined by using the variational radial wave functions of the initial and the final state and explicitly performing the angular integration given by[36]

$$
\Gamma_{f i}=\frac{4 \alpha}{9}\left(\frac{e_{Q} m_{\bar{q}}-e_{\bar{q}} m_{Q}}{m_{\bar{q}}+m_{Q}}\right)^{2} k^{3}|\langle f|r| i\rangle|^{2} \frac{E_{f}}{M_{i}} \times \begin{cases}1 & \text { for }{ }^{3} P_{J} \rightarrow{ }^{3} S_{1} \\ 1 & \text { for }{ }^{1} P_{1} \rightarrow{ }^{1} S_{0} \\ (2 J+1) / 3 & \text { for }{ }^{3} S_{1} \rightarrow{ }^{3} P_{J} \\ 3 & \text { for }{ }^{1} S_{0} \rightarrow{ }^{1} P_{1}\end{cases}
$$

Here, $\alpha$ is the fine structure constant, $k$ is the photon energy, $e_{\bar{q}}$ and $e_{Q}$ are the quark charges in units of the proton charge, $E_{f}$ is the energy of the final meson state, $M_{i}$ is the mass of the initial meson state, and $m_{\bar{q}}$ and $m_{Q}$ are the quark masses employed within the present work.

The $\mathrm{E} 1$ radiative transition widths are listed in tables (4). 


\subsection{Magnetic Dipole Transitions}

The M1 rate for transitions between $S$-wave levels is given by [37, 38]

$$
\Gamma_{M 1}(i \rightarrow f+\gamma)=\frac{16 \alpha}{3} \mu^{2} k^{3}\left(2 J_{f}+1\right)\left|\left\langle f\left|j_{0}(k r / 2)\right| i\right\rangle\right|^{2},
$$

where the magnetic dipole moment is

$$
\mu=\frac{m_{\bar{q}} e_{Q}-m_{Q} e_{\bar{q}}}{4 m_{\bar{q}} m_{Q}}
$$

and $k$ is the photon energy. Rates for the allowed transitions between the spin-triplet and the spinsinglet states are given in Table (4).

Table 4. Electromagnetic transition widths in the $B$ meson.

\begin{tabular}{ccc}
\hline Transition & $k(\mathrm{MeV})$ & $\Gamma(\mathrm{keV})$ \\
\hline $1^{3} P_{2} \rightarrow 1^{3} S_{1} \gamma$ & 0.419 & 79.98 \\
$1 P_{1}^{\prime} \rightarrow 1^{3} S_{1} \gamma$ & 0.411 & 14.99 \\
$1 P_{1}^{\prime} \rightarrow 1^{1} S_{0} \gamma$ & 0.472 & 91.72 \\
$1 P_{1} \rightarrow 1^{1} S_{0} \gamma$ & 0.470 & 22.40 \\
$1 P_{1} \rightarrow 1^{3} S_{1} \gamma$ & 0.409 & 59.60 \\
$1^{3} P_{0} \rightarrow 1^{3} S_{1} \gamma$ & 0.385 & 61.91 \\
$2{ }^{3} S_{1} \rightarrow 1^{3} P_{2} \gamma$ & 0.114 & 4.16 \\
$2^{3} S_{1} \rightarrow 1^{3} P_{0} \gamma$ & 0.150 & 1.89 \\
$2^{1} S_{0} \rightarrow 1 P_{1}^{\prime} \gamma$ & 0.111 & 5.51 \\
$2^{1} S_{0} \rightarrow 1 P_{1} \gamma$ & 0.113 & 1.45 \\
$2^{3} S_{1} \rightarrow 1 P_{1}^{\prime} \gamma$ & 0.123 & 0.611 \\
$2^{3} S_{1} \rightarrow 1 P_{1} \gamma$ & 0.125 & 2.61 \\
\hline $1^{3} S_{1} \rightarrow 1^{1} S_{0} \gamma$ & 0.066 & 0.314 \\
$2^{3} S_{1} \rightarrow 2^{1} S_{0} \gamma$ & 0.012 & 0.002 \\
$3^{3} S_{1} \rightarrow 3^{1} S_{0} \gamma$ & 0.005 & 0.000 \\
$2^{3} S_{1} \rightarrow 1^{1} S_{0} \gamma$ & 0.585 & 41.087 \\
$2{ }^{1} S_{0} \rightarrow 1^{3} S_{1} \gamma$ & 0.514 & 85.120 \\
\hline
\end{tabular}

\section{Conclusion}

The mass spectra, decay constants as well as electromagentic transition widths have been obtained for the $B$ meson within potential model scheme. It can be observed from table 1 that our predictions for the spin-averaged masses are in good agreement with experimental as well as other theoretical estimates. The complete mass spectrum is in general good agreement with experimental measurements as well as with other theoretical predictions as can be seen from table 2. Our prediction for the mass of the $1^{3} P_{0} ; 5.729 \mathrm{GeV}$ is fairly close the experimental value $5.732_{ \pm 0.02}^{ \pm 0.005} \mathrm{GeV}$. From table 3 it is found that our predictions for the vector and pseudoscalar decay constants are in general overestimated when compared with estimates from other theoretical models. Due to scarcity of reliable experimental estimates for E1 and M1 transition rates the validity of estimates obtained in the present work cannot be justified. 


\section{References}

[1] J. Beringer, P.D. Group, Phys. Rev. D86, 010001 (2012)

[2] D. Ebert, R. Faustov, V. Galkin, Eur. Phys. J. C66, 197 (2010)

[3] M. Di Pierro, E. Eichten, Phys. Rev. D 64, 114004 (2001)

[4] J. Zeng, J.W. Van Orden, W. Roberts, Phys. Rev. D 52, 5229 (1995)

[5] S. Godfrey, R. Kokoski, Phys. Rev. D 43, 1679 (1991)

[6] S.N. Gupta, J.M. Johnson, Phys. Rev. D 51, 168 (1995)

[7] T. Lahde, C. Nyfalt, D. Riska, Nucl. Phys. A674, 141 (2000), hep-ph/9908485

[8] N. Devlani, A. Rai, Eur. Phys. J. A50, 154 (2014)

[9] N. Devlani, A.K. Rai, Phys. Rev. D 84, 074030 (2011)

[10] N. Devlani, A. Rai, Eur. Phys. J. A48, 104 (2012)

[11] N. Devlani, A. Rai, Int. J. Theor. Phys. 52, 2196 (2013)

[12] Y. Koma, M. Koma, H. Wittig, Phys. Rev. Lett 97, 122003 (2006)

[13] A.K. Rai, R.H. Parmar, P.C. Vinodkumar, J. Phys. G: Nucl. Part. Phys. 28, 2275 (2002)

[14] A.K. Rai, J.N. Pandya, P.C. Vinodkumar, J. Phys. G: Nucl. Part. Phys. 31, 1453 (2005)

[15] A.K. Rai, B. Patel, P.C. Vinodkumar, Phys. Rev. C 78, 055202 (2008)

[16] A.M. Badalian, A.I. Veselov, B.L.G. Bakker, Phys. Rev. D 70, 016007 (2004)

[17] Y.A. Simonov, Physics of Atomic Nuclei 58, 107 (1995)

[18] D. Ebert, R.N. Faustov, V.O. Galkin, Phys. Rev. D 79, 114029 (2009)

[19] D.S. Hwang, G.H. Kim, Phys. Rev. D 55, 6944 (1997)

[20] B. Patel, P.C. Vinodkumar, J. Phys. G. 36, 035003 (2009)

[21] E.J. Eichten, C. Quigg, Phys. Rev. D 49, 5845 (1994)

[22] D. Gromes, Z. Phys. C26, 401 (1984)

[23] S. Gershtein, V. Kiselev, A. Likhoded, A. Tkabladze, Phys. Usp. 38, 1 (1995)

[24] D. Ebert, R. Faustov, V. Galkin, Eur. Phys. J. C71, 1825 (2011)

[25] Y.b. Dai, C.S. Huang, H.Y. Jin, Phys. Lett. B331, 174 (1994)

[26] R. Van Royen, V. Weisskopf, Nuovo Cim. A50, 617 (1967)

[27] E. Braaten, S. Fleming, Phys. Rev. D 52, 181 (1995)

[28] H. Na, C.J. Monahan, C.T. Davies, R. Horgan, G.P. Lepage et al. (2012), arXiv:hep-lat/1202.4914

[29] E. Neil et al. (Fermilab Lattice Collaboration, MILC Collaboration), PoS LATTICE2011, 320 (2011), arXiv:hep-lat/1112.3978

[30] D. Asner et al. (Heavy Flavor Averaging Group) (2010), arXiv: hep-ex/1010 . 1589

[31] M.Z. Yang, Eur. Phys. J. C72, 1880 (2012), arXiv: hep-ph/1104 . 3819

[32] C. Albertus, E. Hernández, J. Nieves, J.M. Verde-Velasco, Phys. Rev. D 71, 113006 (2005)

[33] K. Bowler et al. (UKQCD Collaboration), Nucl.Phys. B619, 507 (2001), hep-lat/0007020

[34] A. Abd El-Hady, A. Datta, J.P. Vary, Phys. Rev. D 58, 014007 (1998)

[35] D.S. Hwang, G.H. Kim, Phys. Rev. D 55, 6944 (1997)

[36] S.F. Radford, W.W. Repko, M.J. Saelim, Phys. Rev. D 80, 034012 (2009)

[37] D.M. Li, P.F. Ji, B. Ma, Eur. Phys. J. C71, 1 (2011)

[38] N. Brambilla, S. Eidelman, B. Heltsley, R. Vogt, G. Bodwin et al., Eur. Phys. J. C71, 1534 (2011) 